\title{
Momento da Chuva após a Aplicação e a Eficácia dos Herbicidas Sulfosate E GLYPHOSATE Aplicados em Diferentes FormULAÇões ${ }^{1}$
}

\author{
Rainfastness and Glyphosate and Sulfosate Efficacy Using Different Formulations
}

PEDRINHO JÚNIOR, A.F.F. ${ }^{2}$, MARTINI, G. ${ }^{2}$, FELICI, G.V. ${ }^{2}$, PIVA, F.M. ${ }^{2}$ e DURIGAN, J.C. ${ }^{3}$

\begin{abstract}
RESUMO - Com o objetivo de estudar a influência da chuva sobre o desempenho dos herbicidas sulfosate e glyphosate em diferentes formulações, foram conduzidos dois experimentos, um no inverno de 2000 e outro no verão de 2001, na Fazenda Experimental de Ensino e Pesquisa da Faculdade de Ciências Agrárias e Veterinárias, Campus de Jaboticabal-SP. Os experimentos foram instalados segundo o delineamento experimental de blocos ao acaso, com quatro repetições, no arranjo fatorial de $4 \times 5+1$, ou seja, quatro tratamentos de herbicidas, cinco períodos sem chuva após a aplicação e uma testemunha, que não recebeu chuva. As formulações de glyphosate foram: SAqC (1,0 L ha-1), GrDA $\left(0,5 \mathrm{~kg} \mathrm{ha}^{-1}\right)$, SAqC Transorb $\left(0,75 \mathrm{~L} \mathrm{ha}^{-1}\right)$, mais o sulfosate SAqC (1,09 L ha-1). Os períodos sem chuva após a aplicação foram de 1, 2, 4, 6 e $>48$ horas. Os herbicidas foram aplicados em pós-emergência das plantas daninhas, utilizandose de um pulverizador costal, à pressão constante (mantida por ar comprimido) de $30 \mathrm{lbf} \mathrm{pol}^{-2}$. A chuva foi simulada com um sistema de irrigação por aspersão. A lâmina de água variou entre 18 e $19 \mathrm{~mm}$. Em ambas as épocas, a chuva simulada foi prejudicial à ação dos herbicidas, principalmente quando feita nos menores intervalos após a aplicação. Os sintomas de fitointoxicação apareceram mais rapidamente no verão. A formulação Transorb, comercializada como não sendo afetada pela chuva uma hora após a aplicação, não teve o desempenho esperado, tanto no inverno quanto no verão, para períodos de até seis horas sem chuva após a aplicação. $\mathrm{O}$ sulfosate apresentou o melhor controle geral das plantas avaliadas, quando se simulou chuva após seis horas, em ambas as épocas. A formulação GrDA foi a mais afetada pela ação da chuva em ambas as épocas.
\end{abstract}

Palavras-chave: controle de plantas daninhas, chuva simulada, glyphosate, sulfosate.

\begin{abstract}
Two field assays were carried out to evaluate the rainfall effects on sulfosate and glyphosate herbicide activity, using different formulations, under two growing seasons (winter and summer). The experiments were conducted at the FCAV/UNESP Experimental Station, Jaboticabel, Brazil, during 2000/01. The formulations were glyphosate SA (1.0 L ha $\left.\mathrm{C}^{-1}\right), W G\left(0.5 \mathrm{~L} \mathrm{ha}^{-1}\right)$, Transorb $\left(0.75 \mathrm{~L} \mathrm{ha}^{-1}\right)$ and sulfosate $\mathrm{SA}\left(1.09 \mathrm{~L} \mathrm{ha}^{-1}\right)$. In the field, all the trials were arranged in a randomized block experimental design, with four replications. The treatments were arranged in factorial design $5 \times 4+1$ factors, with five periods between the herbicide spraying and the rain incidence $(1,2,4$, 6 and $>48 \mathrm{~h}$ ), plus one control treatment. The herbicide treatments were applied using a back spraying, with six nozzles DG 11002, spaced by $0.5 \mathrm{~m}$, at 30 p.s.i. constant pression. Artificial rain was provided by a top irrigation system raining $18-19 \mathrm{~mm}$ during one hour. The artificial rain reduced glyphosate action, mainly in the shorter periods between herbicide application and the rain incidence. The herbicide symptoms appeared quicker in the summer trials. The Transorb formulation (advertised as not affected by rain one hour after spraying) had the phytointoxication symptoms reduced by the rain up to six hours after spraying, both in the summer and winter. The herbicide sulfosate promoted better control when the rain occurred six hours after spraying, in both seasons.
\end{abstract}

Key words: weed control, winter and summer, rainfastness, glyphosate, sulfosate.

1 Recebido para publicação em 30/7/2001 e na forma revisada em 15/2/2002.

Trabalho de graduação do primeiro autor, <aimarpjr@zipmail.com.br>.

2 Graduandos em Agronomia, FCAV/UNESP, Rod. Paulo Castellani Km 5, 14870-000 Jaboticabal-SP; ${ }^{3}$ Prof. Titular, FCAV/ UNESP, Jaboticabal-SP.

Planta Daninha, Viçosa-MG, v.20, n.1, p.115-123, 2002 


\section{INTRODUÇÃO}

A interferência das plantas daninhas é um dos principais fatores responsáveis pela redução de produtividade de diversas culturas. Esse prejuízo ocorre, principalmente, devido à competição por luz, água, nutrientes e $\mathrm{CO}_{2}$ (Blanco et al., 1973), trazendo sérios prejuízos financeiros para os agricultores, que perdem em produtividade da cultura e qualidade do produto final.

Um dos grupos de herbicidas mais vendidos no mundo é o dos inibidores de EPSPs, no qual estão o glyphosate e o sulfosate, com maior participação do primeiro no mercado, pois são mais de 90 marcas comerciais com esse ingrediente ativo. Os inibidores de EPSPs perfazem $12 \%$ da venda global de herbicidas, sendo distribuídos em 119 países, com mais de 150 marcas comerciais (Heap, 1997). Atualmente, um dos herbicidas mais utilizados no Brasil é o glyphosate, devido ao seu preço e à sua eficácia para grande número de espécies de plantas daninhas. O seu consumo só não é maior devido à não-seletividade às plantas anuais cultivadas, o que limita sua utilização em pósemergência dessas culturas.

O glyphosate transloca-se nas plantas, matando as suas estruturas reprodutivas, como rizomas, bulbos e tubérculos. Possui um coeficiente de partição octanol/água extremamente baixo, indicando que a molécula tem baixa afinidade por substâncias graxas e altíssima por água. Essa é uma das causas principais de sua absorção relativamente lenta, tornando a aplicação arriscada em épocas chuvosas e obrigando o fabricante a recomendar período sem chuvas de seis horas após a aplicação (Kruse et al., 2000). Outros fatores ambientais também podem influenciar a eficácia do herbicida para o controle de plantas daninhas, como a temperatura, a luminosidade, a umidade do solo, a umidade relativa do ar e a geada (Baird, 1971; Fawcett \& Davis, 1976; Fernandez, 1979).

Vários trabalhos são realizados com o objetivo de estudar as razões dos possíveis aumentos e reduções na eficácia do glyphosate para o controle de plantas daninhas (Durigan, 1993). Novas formulações, mais concentradas e adicionadas de adjuvantes, estão sendo testadas. Estudos têm sido realizados para que as recomendações possam garantir maior segurança ao agricultor no momento da aplicação do herbicida, mesmo em época chuvosa. A reaplicação é prática que onera os custos de produção, demanda mais tempo de trabalho e aumenta a poluição ambiental; além disso, a eficácia pode ficar comprometida pelo estresse das plantas causado pelo herbicida aplicado anteriormente.

O presente trabalho teve por objetivo estudar a influência da chuva, realizada em diferentes horas após a aplicação do herbicida sulfosate e do glyphosate em diferentes formulações, no inverno e no verão.

\section{MATERIAL E MÉTODOS}

Dois experimentos, um no inverno e outro no verão, foram conduzidos na Fazenda Experimental de Ensino e Pesquisa da Faculdade de Ciências Agrárias e Veterinárias - UNESP, Campus de Jaboticabal-SP, durante o período de junho-agosto de 2000 e de janeiro-março de 2001.

O clima da região, segundo a classificação de Koppen, é do tipo Cwa, ou seja, mesotérmico de inverno seco, com temperatura média do mês mais quente em torno de $22{ }^{\circ} \mathrm{C}$ e do mês mais frio, de $18^{\circ} \mathrm{C}$. A cidade de Jaboticabal está localizada na latitude de $21^{\circ} 15^{\prime} 22^{\prime}$ e na longitude de $48^{\circ} 18^{\prime} 58^{\prime \prime}$, com altitude de $580 \mathrm{~m}$. O solo do local foi classificado, segundo Centurion (1998), como Latossolo Vermelho-Escuro, eutrófico, horizonte A moderado, textura muito argilosa e relevo suavemente ondulado.

Os experimentos foram instalados segundo o delineamento experimental de blocos ao acaso, com quatro repetições, no arranjo fatorial $4 \times 5+1$. Os quatro herbicidas constituíram sempre o fator A, e os cinco períodos livres de chuva após a aplicação representaram o fator B. As parcelas experimentais mediram $3,0 \mathrm{~m}$ de largura e 3,5 m de comprimento, no experimento de inverno, e 3,0 x 4,0 m, no de verão.

Os herbicidas foram aplicados em pósemergência das plantas daninhas, utilizandose um pulverizador costal, à pressão constante (mantida por ar comprimido) de $30 \mathrm{lbf} \mathrm{pol}^{-2}$, munido de barra com seis bicos DG 11002 , espaçados de $0,50 \mathrm{~m}$, perfazendo uma faixa de aplicação com 3,0 m de largura. 
Na Tabela 2 são apresentadas as plantas daninhas predominantes, os estádios de desenvolvimento e a intensidade da infestação, durante as aplicações dos herbicidas, em cada um dos experimentos. Os dados referentes às condições climáticas, nos diferentes horários de aplicação e épocas do ano, para cada experimento, são apresentados na Tabela 3.

Nos dois experimentos, a chuva foi simulada com o auxílio de um sistema de irrigação por aspersão, e a lâmina de água variou entre 18 e $19 \mathrm{~mm}$, molhando-se todas as parcelas de uma só vez.

Tabela 1 - Formulações do herbicida glyphosate, doses e intervalos entre a aplicação e a chuva simulada. Jaboticabal-SP, 2000/2001

\begin{tabular}{|c|c|c|}
\hline Formulações & Dose (L ou kg ha ${ }^{-1}$ do p.c.) & $\begin{array}{c}\text { Intervalos, em horas, entre a aplicação } \\
\text { e a chuva simulada }\end{array}$ \\
\hline Glyphosate SAqC $^{(\mathrm{a} 1)}\left(360\right.$ g i.a. $\left.\mathrm{L}^{-1}\right)$ & 1,00 & $1,2,4,6$ e $>48$ \\
\hline Glyphosate GrDA $^{(\text {b) }}\left(720\right.$ g i.a. $\left.L^{-1}\right)$ & 0,50 & $1,2,4,6 \mathrm{e}>48$ \\
\hline Glyphosate SAqC $^{(\mathrm{a} 2)}\left(480\right.$ g i.a. $\left.\mathrm{L}^{-1}\right)$ & 0,75 & $1,2,4,6 \mathrm{e}>48$ \\
\hline Glyfosate $\mathrm{SAqC}^{(\mathrm{c})}\left(330 \mathrm{~g}\right.$ i.a. $\left.\mathrm{L}^{-1}\right)$ & 1,09 & $1,2,4,6$ e $>48$ \\
\hline Testemunha & sem herbicida & -- \\
\hline
\end{tabular}

p.c.- produto - comercial (a1) Roundup SAQC, (a2) RoundupTransorb; (b) RoundupWG; (c) Zapp.

Tabela 2 - Plantas daninhas predominantes, estádios de desenvolvimento e intensidade de infestação no momento da aplicação dos herbicidas, em cada um dos experimentos, nas duas épocas do ano. Jaboticabal-SP, 2000/2001

\begin{tabular}{|c|l|l|c|}
\hline \multirow{2}{*}{ Épocas do ano } & \multicolumn{1}{|c|}{ Planta Daninha } & \multicolumn{1}{|c|}{ Estágio de desenvolvimento } & $\begin{array}{c}\text { Intensidade de } \\
\text { infestação (\%) }\end{array}$ \\
\hline \multirow{2}{*}{ Inverno } & Amaranthus retroflexus & $0,4-0,6 \mathrm{~m}$ de altura (com inflorescência) & 40 \\
& Brachiaria plantaginea & $0,25 \mathrm{~m}$ de altura (não florescida) & 35 \\
\hline \multirow{2}{*}{ Verão } & Alternanthera tenella & $0,5 \mathrm{~m}$ de altura (em pleno florescimento) & 25 \\
& Brachiaria plantaginea & $0,6 \mathrm{~m}$ de altura (com sementes) & 65 \\
\hline
\end{tabular}

Tabela 3 - Dados climáticos obtidos nos respectivos horários de aplicação do herbicida, em cada um dos experimentos. Jaboticabal - SP, 2000/2001

\begin{tabular}{|c|c|c|c|c|c|c|c|}
\hline \multirow{2}{*}{ Épocas do ano } & Aplicação & \multirow{2}{*}{$\begin{array}{c}\text { Horário de } \\
\text { aplicação (h) }\end{array}$} & \multirow{2}{*}{$\begin{array}{l}\text { UR do ar } \\
\quad(\%)\end{array}$} & \multirow{2}{*}{$\begin{array}{c}\mathrm{T}^{\circ} \mathrm{C} \\
\text { Ambiente }\end{array}$} & \multirow{2}{*}{$\begin{array}{c}\mathrm{T}^{\circ} \mathrm{C} \text { Solo } \\
(5 \mathrm{~cm})\end{array}$} & \multirow{2}{*}{$\begin{array}{l}\text { Vel. vento } \\
(\mathrm{km} / \text { hora })\end{array}$} & \multirow{2}{*}{$\begin{array}{c}\text { Nebulosidade } \\
(\%)\end{array}$} \\
\hline & Dia/mês/ano & & & & & & \\
\hline \multirow{5}{*}{ Inverno } & \multirow{5}{*}{$14 / 6 / 2000$} & $8 \mathrm{~h} 5-8 \mathrm{~h} 20$ & 75 & 27,0 & 16,5 & Ausente & 5 \\
\hline & & $9 \mathrm{~h} 55-10 \mathrm{~h} 10$ & 50 & 30,5 & 18,2 & 4,00 & 0 \\
\hline & & $11 \mathrm{~h} 55-12 \mathrm{~h} 10$ & 42 & 33,0 & 20,8 & 5,20 & 0 \\
\hline & & $12 \mathrm{~h} 55-13 \mathrm{~h} 10$ & 44 & 32,0 & 21,8 & 4,87 & 0 \\
\hline & & $16 \mathrm{~h} 30-16 \mathrm{~h} 45$ & 41 & 30,0 & 22,2 & Ausente & 5 \\
\hline \multirow{5}{*}{ Verão } & \multirow{5}{*}{$4 / 1 / 2001$} & $8 \mathrm{~h} 20-8 \mathrm{~h} 40$ & 70 & 27,0 & 23,0 & 3,0 & 70 \\
\hline & & $10 \mathrm{~h} 20-10 \mathrm{~h} 40$ & 79 & 31,0 & 22,0 & 5,4 & 85 \\
\hline & & $12 \mathrm{~h} 25-12 \mathrm{~h} 45$ & 65 & 33,0 & 24,0 & 3,5 & 20 \\
\hline & & $13 \mathrm{~h} 30-13 \mathrm{~h} 50$ & 61 & 32,0 & 24,0 & 1,2 & 50 \\
\hline & & $17 \mathrm{~h} 30-17 \mathrm{~h} 50$ & 67 & 27,0 & 23,5 & Ausente & 70 \\
\hline
\end{tabular}


As diferenças de tempo entre as chuvas e as aplicações de herbicidas foram conseguidas antecipando-se estas últimas em seis, quatro, duas e uma hora. O período superior a 48 horas sem chuva foi conseguido realizando-se a aplicação dos herbicidas, em seus respectivos tratamentos, após uma hora do final da simulação da chuva, quando as plantas apresentavam as lâminas foliares totalmente secas. Após esta última aplicação, monitorou-se o índice pluviométrico do local onde se encontrava instalado o ensaio por 48 horas, com o objetivo de registrar possiveis ocorrências pluviométricas, porém não foi registrada nenhuma, em ambas as épocas.

Foram realizadas avaliações visuais da eficácia dos herbicidas aos 17, 26, 33 e 41 dias após a aplicação (DAA), no inverno, e aos 7, 14, 21 e 33 DAA, no verão. Em todas as avaliações, foram atribuídas, visualmente, as porcentagens de controle para as duas principais espécies de plantas daninhas, em que nenhum controle equivale à nota 0 e controle total, à nota 100. Além das avaliações gerais da comunidade infestante, também foram quantificadas as massas das plantas recém-colhidas aos 41 DAA e posteriormente secas. Para isso, as plantas foram cortadas rente ao solo, em cada parcela, utilizando-se de um quadrado para amostragem de 0,75 x 0,75 m. Logo após a coleta, pesou-se o material coletado, no local, para obter os dados de massa recém-colhida. A seguir, o material foi colocado em sacos de papel e levado para estufa de circulação forçada de ar, a $65{ }^{\circ} \mathrm{C}$, onde permaneceu por quatro dias. Em seguida, as amostras foram pesadas, para obtenção dos dados de massa seca.

Os dados foram submetidos à análise de variância, as quais foram realizadas com o auxílio do programa ESTAT, com as porcentagens transformadas em arco sen $\sqrt{ } \%$.

\section{RESULTADOS E DISCUSSÃO}

No inverno, nenhum herbicida apresentou resultado satisfatório de controle até os 26 dias após a aplicação (DAA), mesmo quando livre de chuva por um período superior a 48 horas. Pelos dados da Tabela 4, pode-se constatar que nas primeiras avaliações (17 e 26 DAA) não existiram interações significativas entre os tratamentos com herbicidas e os períodos sem chuva após a aplicação. Níveis de controle satisfatórios apareceram somente aos 41 DAA, nos tratamentos que receberam chuva em período superior a 48 horas. Nesta condição, o glyphosate Transorb apresentou melhor controle $(91,2 \%)$, seguido de glyphosate Saqc $(87,5 \%)$ e glyphosate GRDA (85\%), ficando o herbicida sulfosate com o pior controle nesse período. No entanto, nos tratamentos que receberam chuva após seis horas da aplicação, o sulfosate, apesar de não proporcionar controle satisfatório, ficou à frente dos demais herbicidas. Alguns autores, como Devine et al. (1983) e Masiunas \& Weller (1988), afirmam que o controle inicial de glyphosate é mais rápido quando submetido a temperaturas entre 21 e $28{ }^{\circ} \mathrm{C}$. Esses autores relatam que o menor efeito constatado em temperaturas menores $\left(10\right.$ a $\left.13^{\circ} \mathrm{C}\right)$ deve-se à menor absorção do herbicida, pelo fato de a planta encontrarse com seu metabolismo diminuído. Entretanto, Baird \& Begeman (1972) e Caseley (1972) chegaram a resultados que diferem dos autores anteriores: estudando o controle de Agropyron repens pelo herbicida glyphosate, constataram maior eficácia a $16{ }^{\circ} \mathrm{C}$ do que a $32{ }^{\circ} \mathrm{C}$.

Harker \& Dekker (1988) estudaram o efeito da temperatura nos herbicidas cloproxydim, quizalofop-ethyl, glyphosate, sethoxydim, fluazifop-butyl e haloxyfop-methyl, constatando que todos se comportaram de maneira diferenciada no que se refere ao controle de Agropyron repens. Na maioria dos casos, a maior translocação esteve associada às temperaturas mais elevadas.

Outro fator importante para o desempenho do glyphosate é a umidade relativa do ar, que, no inverno do centro-oeste paulista, é mais baixa que no verão. Nilsson \& Hallgren (1989) verificaram que a eficácia do glyphosate no controle de Elymus repens foi maior quando a umidade relativa do ar era de $90 \%$, em relação a $55 \%$.

Aos 26 DAA, as médias de controle da comunidade infestante variaram de 18,6\% (glyphosate GrDA) a 26,7\% (glyphosate SAqC Transorb), mas não diferiram significativamente. Nessa mesma data, a média de controle no tratamento com chuva após uma hora foi de $10 \%$; para seis horas, foi de $13,2 \%$; e somente onde a chuva ocorreu em período superior a 48 horas atingiu-se $70,9 \%$ de controle. 
Tabela 4 - Resultados do teste F aplicado às médias das porcentagens de controle geral das plantas daninhas, influenciadas por chuvas simuladas em diferentes períodos após a aplicação de formulações do herbicida glyphosate. Jaboticabal-SP, 2000

\begin{tabular}{|c|c|c|c|c|c|}
\hline \multirow{2}{*}{\multicolumn{2}{|c|}{ Fatores }} & \multicolumn{4}{|c|}{ Dias Após a Aplicação } \\
\hline & & 17 & 26 & 33 & 41 \\
\hline \multicolumn{2}{|l|}{$\begin{array}{l}\text { Testemunha x Fatores } \\
\text { Herbicidas }(\mathrm{H}) \\
\text { Período sem chuva }(\mathrm{P}) \\
\text { F x P }\end{array}$} & $\begin{array}{r}87,41^{* *} \\
7,82^{* *} \\
233,45^{* *} \\
0,85^{\mathrm{NS}}\end{array}$ & $\begin{array}{r}90,27^{* *} \\
4,05^{\mathrm{NS}} \\
195,85^{* *} \\
0,52^{\mathrm{NS}}\end{array}$ & $\begin{array}{r}403,95 * * \\
9,76 * * \\
386,05 * * \\
8,30 * *\end{array}$ & $\begin{array}{r}456,60 * * \\
8,58 * * \\
426,30 * * \\
7,35 * *\end{array}$ \\
\hline $\mathrm{CV}(\%)$ & & 21,91 & 21,6 & 10,2 & 9,6 \\
\hline Herbicidas & $\begin{array}{l}\text { glyphosate SaqC } \\
\text { glyphosate GrDA } \\
\text { glyphosate SAqC Transorb } \\
\text { sulfosate SaqC }\end{array}$ & $\begin{array}{l}(\mathrm{a}) 18,4 \mathrm{~B}(14,1)^{(\mathrm{b})} \\
18,6 \mathrm{~B}(15,1) \\
20,2 \text { B }(16,1) \\
24,2 \text { A }(19,8)\end{array}$ & $\begin{array}{l}20,8 \text { A }(22,5) \\
23,3 \text { A }(18,6) \\
21,6 \text { А }(26,7) \\
23,9 \text { А }(24,6)\end{array}$ & & \\
\hline $\mathrm{dms}$ a $5 \%$ & & 3,56 & 5,36 & & \\
\hline Períodos sem Chuva (h) & $\begin{array}{l}1 \\
2 \\
4 \\
6 \\
>48\end{array}$ & $\begin{array}{l}10,0 \mathrm{C}(3,7) \\
12,5 \text { ВC }(5,8) \\
13,6 \text { ВC }(6,1) \\
16,5 \text { В }(8,6) \\
49,2 \text { А }(57,1)\end{array}$ & $\begin{array}{l}15,3 \text { C }(10,0) \\
16,8 \text { ВC }(13,0) \\
16,3 \text { ВС }(8,3) \\
20,5 \text { В }(13,2) \\
57,7 \text { А }(70,9)\end{array}$ & & \\
\hline $\mathrm{dms}$ a $5 \%$ & & 4,23 & 5,18 & & \\
\hline
\end{tabular}

(a) Dados transformados em arco sen $\sqrt{ } \%$.

(b) Dados originais.

SAqC - Solução Aquosa Concentrada; GrDA - Grânulos Dispersíveis em Água; SAqC Transorb - Solução Aquosa Concentrada com adjuvante especial.

Na Tabela 5 são apresentadas as médias das porcentagens de controle da interação significativa entre as formulações e os períodos sem chuva após a aplicação, aos 41 DAA. Nesta data, pode-se constatar o efeito da chuva sobre o desempenho dos herbicidas. Para todos eles, o período de seis horas sem chuva não foi suficiente para que ocorresse absorção em quantidades letais a uma boa parte dos indivíduos. As médias de controle obtidas no tratamento sem chuva inicial foram estatisticamente superiores às dos demais. Nessa condição, a formulação do glyphosate SAqC Transorb foi mais eficiente $(91,2 \%)$, seguida de glyphosate $\mathrm{SAqC}(87,5 \%)$ e GrDA (85,0\%), as quais não diferiram significativamente entre si. O sulfosate foi o menos eficiente $(73,7 \%)$, mas foi o herbicida menos afetado pela chuva com seis horas após a aplicação.

Carvalho (1989) obteve resultados parecidos para formulações de bentazon, acifluorfen, fomesafen, lactofen, chlorimuron-ethyl e fluoroglicofen, que foram afetados pela chuva simulada até o maior período livre de chuva testado, ou seja, seis horas após a aplicação dos herbicidas, tanto na dose recomendada como para o dobro dela. Jakelaitis et al. (2001), estudando o efeito da chuva sobre os herbicidas glyphosate Transorb, sulfosate e glyphosate potássico no controle de Digitaria horizontalis, não observaram diferenças de controle para as mesmas condições de aplicação. Os mesmos autores relatam que as formulações de glyphosate SAqC e WG foram mais afetadas pela chuva, em todos os intervalos avaliados, quando comparados aos demais tratamentos.

Os percentuais de controle constatados na última avaliação e nos tratamentos sem chuva foram iguais ou superiores a $85 \%$ para as formulações de glyphosate, mesmo sob influência de temperaturas menores. O sulfosate, nessa situação, proporcionou apenas $73,7 \%$ de controle. 
Tabela 5 - Médias das porcentagens de controle, atribuídas visualmente, aos 41 DAA, no inverno, para o controle geral de plantas daninhas, influenciadas por chuvas em diferentes horas após a aplicação de formulações do herbicida glyphosate. Desdobramento da interação herbicidas x períodos de chuvas. Jaboticabal-SP, 2000

\begin{tabular}{|c|c|c|c|c|c|}
\hline \multirow{2}{*}{ Herbicidas } & \multicolumn{5}{|c|}{ Período sem chuva (h) } \\
\hline & 1 & 2 & 4 & 6 & $>48$ \\
\hline glyphosate SAqC & a $28,2 * \mathrm{BC}(22,5)^{* *}$ & ab 26,5 C $(20,0)$ & b 28,0 BC $(21,9)$ & b 34,3 B $(32,5)$ & ab 69,3 A $(87,5)$ \\
\hline glyphosate GrDA & a 24,6 BC $(17,5)$ & b 21,6 C $\quad(13,7)$ & b 25,6 BC $(18,7)$ & b 30,3 B $(25,0)$ & b 67,2 A $(85,0)$ \\
\hline glyphosate SAqC -Transorb & a $23,7 \mathrm{C} \quad(15,0)$ & a $28,1 \mathrm{BC}(22,5)$ & b 29,0 BC $(23,7)$ & b 33,1 B $(27,5)$ & a $74,4 \mathrm{~A} \quad(91,2)$ \\
\hline sulfosate SAqC & a $26,5 \mathrm{D} \quad(20,0)$ & a $29,1 \mathrm{D} \quad(23,7)$ & a $36,2 \mathrm{C} \quad(35,0)$ & a 43,5 B $(47,5)$ & c 59,1 A $(73,7)$ \\
\hline \multicolumn{6}{|c|}{ dms para médias de períodos dentro de cada formulação $=6,73$} \\
\hline & & 6,32 & & & \\
\hline
\end{tabular}

* Dados transformados em arco sen $\sqrt{ } \%$.

**Dados originais.

Obs.: letras minúsculas do lado esquerdo e no sentido das colunas comparam as formulações dentro de cada período; letras maiúsculas do lado direito e no sentido das linhas comparam os períodos dentro de cada formulação.

SAqC - Solução Aquosa Concentrada; GrDA - Grânulos Dispersíveis em Água SAqC Transorb - Solução Aquosa Concentrada com adjuvante especial.

Na Tabela 6 são apresentadas as médias de controle químico das plantas daninhas, influenciadas por chuvas, em diferentes horas após a aplicação dos herbicidas, durante o verão. Houve interação significativa entre os herbicidas e os períodos livres de chuva apenas na primeira avaliação (7 DAA). Todos os períodos de chuva afetaram o desempenho dos herbicidas nas avaliações feitas aos 14, 21 e 33 DAA, em relação ao tratamento que não recebeu chuva após a aplicação.

Os tratamentos que não foram submetidos a chuva após a aplicação permaneceram melhor em todas as avaliações, diferindo estatisticamente dos demais. Os herbicidas glyphosate Transorb e sulfosate tiveram melhor desempenho que os demais durante todo o experimento. Esses dados estão de acordo com Jakelaitis et al. (2001), que também observaram bom desempenho desses dois herbicidas quando submetidos a chuva após a aplicação. Observase, aos 33 DAA, um resultado considerado satisfatório também para os períodos de quatro $(78,1 \%)$ e seis horas $(81,5 \%)$, porém ainda distante do ideal. É importante esclarecer, com base nos resultados obtidos, que não há suporte para a tese de que o glyphosate Transorb necessite de apenas uma hora sem chuva após a aplicação para ser eficaz, conforme recomendação da firma produtora.
A não-ocorrência de outras interações significativas, para os dados obtidos no decorrer do experimento, pode estar relacionada à temperatura média e umidade relativa do ar relativamente altas no período experimental, proporcionando metabolismo mais intenso e acelerado das plantas, o que facilitaria a absorção e a translocação dos herbicidas dentro delas, potencializando, assim, o efeito destes. No entanto, o efeito deletério da chuva, principalmente nas primeiras horas, ficou caracterizado, pois a quantidade de ingrediente ativo que consegue penetrar nas folhas das plantas antes que ocorra a chuva, evidentemente, não é suficientemente letal para elas.

Carmona (1995) afirma que o sulfosate é mais eficiente no controle de capim-braquiária que o glyphosate. Osipe (1995) não encontrou diferença significativa entre esses herbicidas para o controle de Cynodon dactylon, com as mesmas formulações. Barros (1995) obteve resultado semelhante no controle de capimbraquiária, sem significância entre as formulações de glyphosate e sulfosate e também entre as doses utilizadas. A semelhança de controle entre os herbicidas glyphosate e sulfosate também foi mostrada por Braz \& Braz (1995), quando os testaram para o controle de tiririca e não encontraram diferenças significativas entre os tratamentos. 
Tabela 6 - Resultados do teste F aplicado às médias das porcentagens de controle geral das plantas daninhas, influenciadas por chuvas simuladas em diferentes períodos após a aplicação de formulações do herbicida glyphosate. Jaboticabal-SP, 2001

\begin{tabular}{|c|c|c|c|c|c|}
\hline \multirow{2}{*}{\multicolumn{2}{|c|}{ Fatores }} & \multicolumn{4}{|c|}{ Dias Após Aplicação } \\
\hline & & \multirow{2}{*}{\begin{tabular}{r}
\multicolumn{1}{c}{7} \\
$111,67 * *$ \\
$14,18 * *$ \\
$57,63 * *$ \\
$2,55 * *$ \\
\end{tabular}} & \multirow{2}{*}{$\begin{array}{r}14 \\
222,14^{* *} \\
10,76^{* *} \\
166,11^{* *} \\
1,72^{\mathrm{NS}} \\
\end{array}$} & \multirow{2}{*}{$\begin{array}{r}21 \\
190,74 * * \\
7,62 * * \\
87,16^{* *} \\
1,51^{\mathrm{NS}} \\
\end{array}$} & \multirow{2}{*}{$\begin{array}{r}33 \\
455,74^{* *} \\
13,52^{* *} \\
43,77^{* *} \\
0,84^{\mathrm{NS}} \\
\end{array}$} \\
\hline $\mathrm{F}$ & $\begin{array}{l}\text { Testemunha x Fatores } \\
\text { Herbicidas }(\mathrm{H}) \\
\text { Período sem chuva }(\mathrm{P}) \\
\text { F x P }\end{array}$ & & & & \\
\hline & $\mathrm{CV}(\%)$ & 19,4 & 13,7 & 14,8 & 9,6 \\
\hline Herbicidas & $\begin{array}{l}\text { glyphosate SaqC } \\
\text { glyphosate GrDA } \\
\text { glyphosate SAqC-Transorb } \\
\text { sulfosate SaqC }\end{array}$ & & $\begin{array}{l}\text { (a) } 36,8 \mathrm{~B}(36,9)^{(\mathrm{b})} \\
37,9 \mathrm{~B}(38,0) \\
43,6 \mathrm{~A}(46,7) \\
44,5 \mathrm{~A}(48,7)\end{array}$ & $\begin{array}{l}41,4 \mathrm{~B} \quad(43,7) \\
43,4 \mathrm{~B} \quad(46,3) \\
46,4 \mathrm{AB}(51,5) \\
50,5 \text { A }(57,9)\end{array}$ & $\begin{array}{l}61,3 \text { BC }(74,9) \\
58,5 \mathrm{C} \quad(70,7) \\
65,5 \mathrm{AB}(80,7) \\
69,4 \text { A } \quad(85,9)\end{array}$ \\
\hline Dms a $5 \%$ & & & 4,46 & 6,39 & 4,87 \\
\hline Período sem chuva (h) & $\begin{array}{l}1 \\
2 \\
4 \\
6 \\
>48\end{array}$ & & $\begin{array}{l}25,9 \text { C }(20,0) \\
30,5 \text { C }(26,5) \\
36,6 \text { B }(35,9) \\
40,8 \text { B }(42,8) \\
69,7 \text { A }(87,6)\end{array}$ & $\begin{array}{l}33,6 \text { D } \quad(31,2) \\
35,4 \text { CD }(34,3) \\
41,1 \text { BC }(43,4) \\
46,2 \text { B } \quad(52,0) \\
70,7 \text { A } \quad(88,2)\end{array}$ & $\begin{array}{ll}55,4 \text { C } & (67,1) \\
55,8 \text { C } & (67,5) \\
62,7 \text { B } & (78,1) \\
65,3 \text { B } & (81,5) \\
79,1 \text { A } & (96,0)\end{array}$ \\
\hline Dms a $5 \%$ & & & 5,31 & 6,39 & 5,79 \\
\hline
\end{tabular}

(a) Dados transformados em arco sen $\sqrt{ } \%$.

(b) Dados originais.

SAqC - Solução Aquosa Concentrada; GrDA - Grânulos Dispersíveis em Água; SAqC Transorb - Solução Aquosa Concentrada com adjuvante especial.

Silva (1996), estudando períodos sem chuva, necessários para o controle de capimbraquiária, mostrou que o glyphosate foi tanto mais eficaz quanto maior o intervalo entre a sua aplicação e a primeira precipitação pluviométrica.

Esses resultados estão de acordo com Rodrigues (1994), que relatou ser o glyphosate um produto vulnerável à chuva, sobretudo quando ela ocorre pouco tempo após sua aplicação, não dando condições para que ele vença plenamente a fase ativa no processo de absorção, sendo facilmente lavado, reduzindo assim a quantidade de herbicida que permanece na folha.

Bariuan et al. (1999) mostraram que o glyphosate só não diminui sua afetividade no controle de Cyperus rotundus se o período sem chuva após a aplicação for de 72 horas.

Bryson (1988) constatou que o glyphosate necessitou de um período mínimo de quatro horas sem chuvas após a aplicação para que não houvesse redução na sua eficácia. Mostrou ainda que essa relação depende da intensidade e freqüência da chuva, da espécie, do estado nutricional da planta e do momento da aplicação do herbicida, entre outras coisas.

Na Tabela 7 são apresentados os pesos das massas recém-colhida e seca dos experimentos de inverno (41 DAA) e de verão (33 DAA). Não houve diferença significativa entre as formulações. Os tratamentos que foram submetidos a um período sem chuva superior a 48 horas produziram menor massa, tanto no inverno quanto no verão. No verão, houve diferença estatística entre os períodos sem chuva, com produção de maior quantidade de massa fresca nos tratamentos submetidos à chuva uma e duas horas após a aplicação dos herbicidas, provando assim haver efeito negativo desta na ação dos diferentes herbicidas.

Comparando os resultados de controle e acúmulo de matéria seca obtidos nas duas épocas do ano, percebe-se que a chuva prejudicou 
a eficácia dos herbicidas, tanto no inverno quanto no verão. O que mais se destacou foi o sulfosate SaqC, que proporcionou os maiores níveis de controle entre os tratamentos que receberam chuva após quatro e seis horas da aplicação. O glyphosate SAqC-T não proporcionou bons resultados quando submetido à chuva simulada após uma e duas horas da aplicação, contradizendo assim a afirmativa da empresa fabricante, de que essa formulação necessita apenas de uma hora sem chuva após a aplicação para manifestar todo o seu potencial de controle. Nos dois experimentos, ficou evidente a fragilidade do glyphosate GrDA com relação à chuva simulada após a aplicação. Esta formulação foi prejudicada em todos os tratamentos onde se simulou chuva após a aplicação, até mesmo com o intervalo de seis horas. Esse efeito é confirmado quando se comparam os resultados obtidos com ela, em períodos sem chuva superiores a 48 horas, quando ela não difere estatisticamente das demais. A maior velocidade de ação, assim como o controle final mais consistente das plantas daninhas com o uso do sulfosate, pode ser explicado pela presença na formulação do sal trimetil sulfônico, enxofre e do adjuvante polialquilglicosídeo. Tanto o sal como o enxofre são altamente solúveis em água e higroscópicos, permitindo maior absorção em condições adversas de umidade. $\mathrm{O}$ adjuvante usado na formulação apresenta bom índice de espalhamento da gota, é higroscópico e seletivo à membrana celular, favorecendo a absorção e a difusão do ácido na planta. Por isso, ocorre boa cobertura foliar, maior concentração do produto nas gotas e vida útil mais longa, penetração mais rápida e menor risco de lavagem pela água, resultando em morte mais rápida das plantas daninhas (Zeneca, 1998).

Em relação à época do ano, o frio potencializou o efeito negativo da chuva sobre a eficácia dos herbicidas. Mesmo os tratamentos que foram submetidos a período livre de chuva superior a 48 horas não apresentaram controle satisfatório das plantas daninhas, o que pode ser explicado pelo baixo metabolismo das plantas quando submetidas a temperaturas menores. A temperatura também pode explicar o fato de se obter um controle das plantas daninhas mais rápido no verão do que no inverno. Com maiores temperaturas, o metabolismo das plantas torna-se mais acelerado, facilitando a penetração e translocação dos herbicidas no seu interior.

Tabela 7 - Médias das massas recém-colhidas e secas acumuladas, aos 41 DAA (no inverno) e 33 DAA (no verão), com chuvas simuladas em diferentes períodos após a aplicação de formulações do herbicida glyphosate. Jaboticabal-SP, $2000 / 2001$

\begin{tabular}{|c|c|c|c|c|c|}
\hline \multirow{2}{*}{\multicolumn{2}{|c|}{ Fatores }} & \multicolumn{2}{|c|}{ Massa (kg) Inverno } & \multicolumn{2}{|c|}{ Massa (kg) Verão } \\
\hline & & Fresca & Seca & Fresca & Seca \\
\hline $\mathrm{F}$ & $\begin{array}{l}\text { Testemunha x Fatores } \\
\text { Herbicidas }(\mathrm{H}) \\
\text { Período sem chuva }(\mathrm{P}) \\
\text { F x P }\end{array}$ & $\begin{array}{r}35,55^{* *} \\
1,00^{\mathrm{NS}} \\
9,83^{* *} \\
0,50^{\mathrm{NS}}\end{array}$ & $\begin{array}{r}37,34^{* *} \\
0,95^{\mathrm{NS}} \\
8,87^{* *} \\
0,58^{\mathrm{NS}}\end{array}$ & $\begin{array}{r}282,63^{* *} \\
2,33^{\mathrm{NS}} \\
5,06^{* *} \\
0,52^{\mathrm{NS}}\end{array}$ & $\begin{array}{r}112,35^{* *} \\
2,10^{\mathrm{NS}} \\
1,75^{\mathrm{NS}} \\
0,47^{\mathrm{NS}}\end{array}$ \\
\hline $\mathrm{CV}(\%)$ & & 30,6 & 23,5 & 51,3 & 44,9 \\
\hline Herbicidas & $\begin{array}{l}\text { glyphosate SAqC } \\
\text { glyphosate GrDA } \\
\text { glyphosate SAqC-Transorb } \\
\text { sulfosate SAqC } \\
\end{array}$ & $\begin{array}{l}0,77 \mathrm{~A} \\
0,85 \mathrm{~A} \\
0,73 \mathrm{~A} \\
0,84 \mathrm{~A}\end{array}$ & $\begin{array}{l}0,18 \mathrm{~A} \\
0,21 \mathrm{~A} \\
0,19 \mathrm{~A} \\
0,20 \mathrm{~A}\end{array}$ & $\begin{array}{l}0,52 \mathrm{~A} \\
0,53 \mathrm{~A} \\
0,40 \mathrm{~A} \\
0,35 \mathrm{~A}\end{array}$ & $\begin{array}{l}0,17 \mathrm{~A} \\
0,17 \mathrm{~A} \\
0,13 \mathrm{~A} \\
0,12 \mathrm{~A}\end{array}$ \\
\hline $\mathrm{dms}$ a $5 \%$ & & 0,214 & 0,040 & 0,25 & 0,06 \\
\hline Períodos sem Chuva (h) & $\begin{array}{l}1 \\
2 \\
4 \\
6 \\
>48 \\
\end{array}$ & $\begin{array}{l}0,89 \mathrm{~A} \\
0,86 \mathrm{~A} \\
0,89 \mathrm{~A} \\
0,90 \mathrm{~A} \\
0,44 \mathrm{~B} \\
\end{array}$ & $\begin{array}{l}0,20 \mathrm{~A} \\
0,21 \mathrm{~A} \\
0,21 \mathrm{~A} \\
0,22 \mathrm{~A} \\
0,13 \mathrm{~B} \\
\end{array}$ & $\begin{array}{l}0,55 \mathrm{~A} \\
0,66 \mathrm{~A} \\
0,44 \mathrm{AB} \\
0,45 \mathrm{AB} \\
0,20 \mathrm{~B} \\
\end{array}$ & $\begin{array}{l}0,17 \mathrm{~A} \\
0,19 \mathrm{~A} \\
0,14 \mathrm{~A} \\
0,14 \mathrm{~A} \\
0,12 \mathrm{~A} \\
\end{array}$ \\
\hline dms a $5 \%$ & & 0,250 & 0,048 & 0,30 & 0,07 \\
\hline
\end{tabular}

SAqC - Solução Aquosa Concentrada; GrDA - Grânulos Dispersíveis em Água; SAqC Transorb - Solução Aquosa Concentrada com adjuvante especial. 


\section{LITERATURA CITADA}

BAIRD, D. D. et al. Introduction of a new broad spectrum postemergent herbicide class with utility for herbaceous perennial weed control. In: NORTH CENTRAL WEED CONTROL CONFERENCE, 26, 1971, Bridgeton.

Proceedings... Bridgeton: 1971. p. 64-68.

BAIRD, D. D.; BEGEMAN, G. F. Postemergence characterization of a new quackgrass herbicide. In: NORTH EASTERN WEED SCIENCE SOCIETY, 26, 1972, Bridgeton. Proceedings... Bridgeton: 1972. p. 100-106.

BARIUAN, J. V.; REDDY, K. N.; WILLS, G. Glyphosate injury, rainfastness, absorption, and translocation in purple nutsedge (Cyperus rotundus). Weed Technol., v. 13, p. 112119, 1999.

BARROS, A. C. Evaluation of biological efficiency of the herbicide sulfosate, compared to glyphosate the desiccation of Brachiaria decumbens. Zeneca Agríc., v. 2, p. 43-44, 1995.

BLANCO, H. G. et al. Observações sobre o período crítico em que as plantas daninhas competem com a soja (Glycine $\max ($ L.) Merrill). O Biológico, v. 39, p .31-35, 1973.

BRAZ, L. T.; BRAZ, B. A. Biological efficiency of sulfosate, compared to glyphosate for the control of seedge (Cyperus rotundus L.). Zeneca Agríc., v. 2, p. 41-42, 1995

BRYSON, C. T. Effects or rainfall on foliar herbicides applied to seedling johnsongrass (Sorghum halepense). Weed Technol., v. 2, p. 124-130, 1988.

CARMONA, R. Effect of foliar converage with dust and spray volume in the efficacy of glyphosate and sulfosate in Brachiaria decumbens. Zeneca Agríc., v. 2, p. 17-18, 1995.

CARVALHO, J. C. Fatores que afetam a eficácia dos herbicidas aplicados em pós emergência no controle de espécies de Ipomoea e outras plantas daninhas dicotiledôneas na cultura da soja (Glycine max (L.) Merrill.). Piracicaba: Escola Superior de Agricultura "Luis de Queiroz", 1989. 135p. Dissertação (Mestrado em Agronomia) - Escola Superior de Agricultura "Luis de Queiroz", 1989.

CASELEY, J. The effect of enviromental factors on the performance of glyphosate against Agropiron repens. Proc. British Weed Control Conf., v. 2, p. 641-647, 1972.

CENTURION, J. F. Caracterização e classificação dos solos da Faculdade de Ciências Agrárias e Veterinárias de Jaboticabal. Jaboticabal: Universidade Estadual Paulista, 1998. 84 p. Tese (Livre Docência) - Universidade Estadual Paulista, 1998.
DEVINE, M. D.; BANDEEN, J. D.; MCKERSIE, B. D. Temperature effects on glyphosate absorption, translocation and distribution in quackgrass (Agropyron repens). Weed Sci., v. 31. p. 461-464, 1983.

DURIGAN, J. C. Efeito de adjuvantes na aplicação e eficácia dos herbicidas. Jaboticabal: Fundação de Estudos e Pesquisas em Agronomia, Medicina Veterinária e Zootecnia, 1993. 42p.

FAWCETT, R. S.; DAVIS, H. Effect of enviroment on glyphosate activity in quackgrass. In: NORTH CENTRAL WEED CONTROL CONFERENCE, 31, 1976, Bridgeton. Proceedings... Bridgeton: 1976. p.159-160.

FERNANDEZ, C. H. Product development field observations. Memphis: Tenn, 1979. 30 p. (Bulletin)

HARKER, K. N.; DEKKER, J. Temperature effects on translocation patterns of several herbicides within quackgrass (Agropyron repens). Weed Sci., v. 36, p. 545-552, 1988.

HEAP, I. M. The occurrence of herbicide resistant weeds world wide. Pestic. Sci., v. 51, n. 2, p. 235-243, 1997.

JAKELAITIS, A. et al. Controle de Digitaria horizontalis pelos herbicidas glyphosate, sulfosate e glyphosate potássico submetidos a diferentes intervalos de chuva após a aplicação. Planta Daninha, v. 19, n. 2, p. 279-285, 2001.

KRUSE, N. D.; TREZZI, M. M.; VIDAL, R. A. Herbicidas inibidores da EPSPs: revisão de literatura. R. Bras. Herbic., v. 1, n. 2, p. 139-146, 2000.

MASIUNAS, J. B.; WELLER, S. C. Glyphosate activity in potato (Solanum tuberosum) under different temperature regimes and light levels. Weed Sci., v.3 6, p. 137-140, 1988.

NILSSON, H.; HALLGREN, E. Experiments with Roundup (Glyphosate) for control of Elymus repens at different relative humidities and with dry and wet stands of Elymus repens at application. An experiment in the greenhouse. Swedish Crop Protec. Conf., v. 2, p. 251-256, 1989.

OSIPE, R. Control of Cynodon dactylon through the application of herbicides in postemergence, in different spray volumes. Zeneca Agríc., v. 2, p.45, 1995.

RODRIGUES, J. D. Absorção, translocação e modo de ação de defensivos (glyphosate e alachlor). Botucatu: Universidade Estadual Paulista, 1994. 114 p.

SILVA, M. A. S. Efeitos de períodos sem chuva, dosagens e volumes de aplicação de glyphosate no controle de Brachiaria decumbens Stapf. Botucatu: Universidade Estadual Paulista, 1996. 67p. Dissertação (Mestrado em Agronomia) - Universidade Estadual Paulista, 1996.

ZENECA Brasil. Treinamento de agroquímicos. São Paulo: 1998. 190 p. (não publicado). 